\title{
The Ecological Validity and ApPlication of the Session-RPE Method for Quantifying Training LOADS IN SWIMMING
}

\author{
Lee K. Wallace, Katie M. Slattery, and Aaron J. Coutts \\ School of Leisure, Sport and Tourism, University of Technology, Sydney, Australia
}

\begin{abstract}
Wallace, LK, Slattery, KM, and Coutts, AJ. The ecological validity and application of the session-RPE method for quantifying training loads in swimming. $J$ Strength Cond Res 23(1): 33-38, 2009-There are few practical methods available for evaluating training loads (TL) during swimming. The purpose of this study was to examine the ecological validity of the sessionrating of perceived exertion (RPE) method for quantifying internal $T L$ in competitive swimmers using heart rate (HR)based methods and distance as criterion measures. This study also examined the correspondence between athlete and coach perceptions of internal TL using the session-RPE method. Twelve (six male, six female) well-trained swimmers (mean \pm $S D$ : age $22.3 \pm 3.1$ years, weight $71.8 \pm 11.6 \mathrm{~kg}$, height $175.0 \pm 9.0 \mathrm{~cm}$ ) participated in this study. All subjects completed a swimming step test to evaluate individual HR zones and blood lactate profile before undertaking 20 swim training sessions where RPE, HR, and distance covered were recorded. Training load was then calculated for each session using the session-RPE, HR-based methods, and session distance. The session-RPE scores were correlated to HR-based methods for measuring internal $\mathrm{TL}$ as well as training distance for each swimmer. All individual correlations between session-RPE, HR-based methods $(r=0.55-0.94 ; p<0.05)$, and distance measures $(r=0.37-0.81 ; p<0.05)$ were significant. Two-way ANOVA showed that there was a significant interaction for training intensity $\times$ coach-athlete perception, indicating that coach RPE was lower than athlete RPE for low-intensity sessions and higher than athlete RPE at high-intensity sessions. The results of this study suggest that session-RPE may provide a practical, noninvasive method for quantifying internal TL in competitive swimmers.
\end{abstract}

KEY WoRDs swimmers, training load, monitoring

Address correspondence to Lee Wallace, lee.k.wallace@uts.edu.au.

23(1)/33-38

Joumal of Strength and Conditioning Research

(C) 2009 National Strength and Conditioning Association

\section{INTRODUCTION}

here are a variety of monitoring methods used by coaches to measure the physical training loads (TLs) undertaken by athletes. However, in sports such as swimming, few valid and reliable methods are available to evaluate the TL undertaken. Typically, the majority of training programs are prescribed using a measure of external TL. External TL is defined as the work completed by an athlete measured independently of his or her internal characteristics. For example, in swimming, coaches often prescribe training with reference to external measures (i.e., distance swum $[\mathrm{m}]$ and/or swimming velocity $\left[\mathrm{m} \cdot \mathrm{s}^{-1}\right]-$ e.g., $10 \times 100 \mathrm{~m}$ on 1:40 min:s holding 1:05 min:s). However, it is the relative physiological stress imposed on the athlete (internal TL), and not the external TL completed by the athlete, that determines the stimulus for training adaptation (22). For example, a similar training session prescribed in terms of an internal TL would be based on physiological measures (e.g., $10 \times 100 \mathrm{~m}$ on 1:40 min:s holding $90 \%$ of maximal heart rate [HRmax]).

Currently, the most widely used methods for evaluating internal TL use heart rate (HR) information as a measure of exercise intensity $(3,11,18,19)$. However, the application of $\mathrm{HR}$ as a measure of exercise intensity in swimming has several limitations. For example, the HR response can be a poor method for evaluating intensity during high-intensity exercise such as weight, interval, intermittent, and plyometric training (14). Many of these training methods are regularly implemented in swimming programs. Furthermore, HR monitoring devices often incur technical failure in water, and manual pulse palpitation requires interruption in exercise.

The session-rating of perceived exertion (RPE) method was proposed by Foster et al. (15) as a practical tool for evaluating internal TL in athletes. This method requires subjects to subjectively rate the intensity of the entire training session using an RPE according to the category ratio scale (CR-10 scale) developed by Borg et al. (5). This intensity value is then multiplied by the total duration (minutes) of the training session to create a single measure of internal TL in arbitrary units (AUs). Previous investigations have shown session-RPE to compare favorably with more complicated methods of quantifying training load in endurance (14), team sport $(7,17)$, and resistance trained athletes (9). 
On the basis of the collective research, it seems that sessionRPE may provide a suitable method for evaluating internal TL in swimming. However, no study has examined its validity and application in competitive swimmers. Therefore, the purpose of this investigation was to compare the session-RPE method with traditional HR-based (internal TL) and distance (external TL) measures for evaluating physical TL in competitive swimmers. Furthermore, this investigation will also examine the correspondence between athletes' and coaches' perceptions of internal TL using the session-RPE method.

\section{Methods}

\section{Experimental Approach to the Problem}

To date, no study has previously measured the ecological validity of the session-RPE method for quantifying internal $\mathrm{TL}$ in swimming. Additionally, to our knowledge, only one study has previously compared coaches' and athletes' perceptions of internal TL using the session-RPE method (16). Therefore, this study will investigate the ecological validity of the session-RPE method for quantifying internal TL in competitive swimmers. To achieve this, the athlete's perception of exercise intensity (RPE) will be compared with commonly used HR methods for quantifying internal TL. Heart rate-based methods were chosen as a criterion measure because HR has previously been established as a valid measure of evaluating exercise intensity (1). Furthermore, this study will also compare the perceptions of internal $\mathrm{TL}$ between athletes and coaches using the session-RPE method.

\section{Subjects}

Twelve (six male, six female) well-trained swimmers (mean \pm $S D$ : age $22.3 \pm 3.1$ years, weight $71.8 \pm 11.6 \mathrm{~kg}$, height $175.0 \pm 9.0 \mathrm{~cm}$ ) volunteered to participate in this study. All subjects had been competing regularly in national competitions for at least 4 years. Six of these subjects were current members of the provincial Institute of Sport Swim Team, and two of the subjects had competed for their country as part of the open national swim team during the previous 12 months. Two qualified swimming instructors also agreed to participate in this study. Preceding the commencement of the study, all subjects were made aware of the potential risks and benefits associated with participation, and written informed consent was obtained by each subject. Ethical approval was granted by the university human research ethics committee for all experimental procedures.

\section{Experimental Protocol}

The present study was completed between December 2005 and March 2006. Each training session was designed and implemented by the swimming instructor with no input from the researcher. After a 1-month familiarization period using the session-RPE method, each subject completed 20 individual training sessions consisting of a variety of different training distances and training intensities.

34 Journal of Strength and Conditioning Research

\section{Physical Training}

All physical training undertaken during this study took place in a 50-m heated pool $\left(27^{\circ} \mathrm{C}\right)$ and was performed as part of the general conditioning phase in the yearly plan. Physical training sessions were designed by the instructors to elicit a variety of training adaptations, with emphasis on improving aerobic conditioning, anaerobic threshold (AT), $\mathrm{VO}_{2} \max$, and speed. The majority of physical training was performed in the form of interval work, where swimming laps were broken down into repeat distances ranging between 25 and $800 \mathrm{~m}$. All physical training sessions lasted between 30 minutes and 2 hours, with swimming distances ranging between 2 and $6 \mathrm{~km}$.

\section{Testing Procedures}

Lactate Threshold Assessment. Each subject completed a swimming step test to establish individual HR zones and blood lactate profiles. The swimming step test consisted of $7 \times$ $200-\mathrm{m}$ efforts, progressing from aerobic swimming to maximal-intensity swimming (20). Immediately after each incremental workload, capillarized blood samples $(30 \mu \mathrm{l})$ were taken from the ear lobe to assess blood lactate concentration $\left(\left[\mathrm{BLa}^{-}\right]\right)$and analyzed using a Lactate Scout Portable Lactate Analyser (Boehringer, Mannheim, Germany). Maximum HR was recorded as the highest HR achieved during the test. The Lactate Analysis macro add-in in Microsoft Excel (SASI, Adelaide, Australia) was used to calculated lactate thresholds (LTs) from fixed $\left[\mathrm{BLa}^{-}\right]$values of 2 and $4 \mathrm{mmol} \cdot \mathrm{L}^{-1}(21)$.

Monitoring Training Load. Daily training load was calculated using the session-RPE method (14). This method involved multiplying the training duration in minutes by the mean training intensity (14). The training intensity was measured by the 10-point Rating of Perceived Exertion Scale (CR-10: RPE) (5) shown in Table 1.

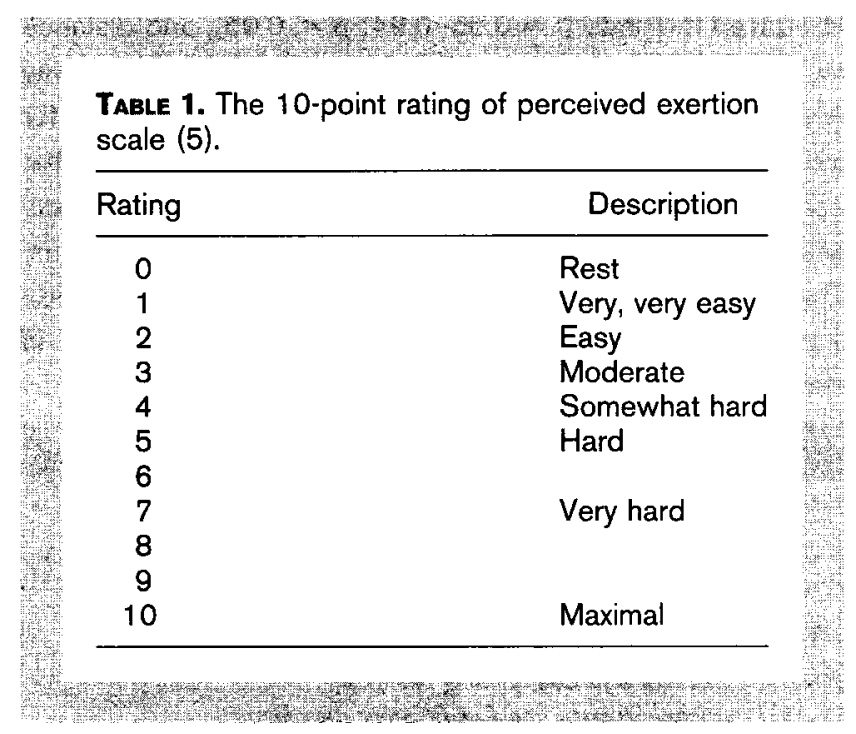


To ensure that each subject reported a global RPE for the entire training session, the RPE was taken 30 minutes after the completion of the session. For comparison of internal TL measures between athlete and coach, individual session-RPE (both RPE and duration) estimates were also taken from the coach before each training session.

Training intensity during each swim training session was also recorded using Polar Team HR monitors (Polar, Kempele, Finland), where HR was recorded every 5 seconds. To reduce HR recording error during training, all subjects were asked to check their HR monitors after each set (approximately 10 minutes). After each training session, HR information was then downloaded to a computer using Polar Advantage Software.

Criterion Methods for Quantifying Physical Training Loads. Several HR-based methods for quantifying internal TL were used as a criterion measure in this investigation. The TRIMP method proposed by Banister (2) was used as the first criterion measure of internal TL and was determined using the following formula:

$$
\text { Training load }=\mathrm{D}(\Delta H R \text { ratio }) \mathrm{e}^{\mathrm{b}(\Delta H R \text { ratio })}
$$

where $\mathrm{D}=$ duration of training session, and $\mathrm{b}=1.67$ for females and 1.92 for males $(4,19)$.

The HR-based method proposed by Edwards (11) was also used as a criterion measure of internal TL in this study. This method involves integrating the total volume with the total intensity of each physical training session relative to five intensity phases. An exercise score for each training bout was calculated by multiplying the accumulated duration in each HR zone by a multiplier allocated to each zone $(50-60 \%$ HRmax $=1,60-70 \%$ HRmax $=2,70-80 \%$ HRmax $=3,80-$ 90\% HRmax $=4$, and $90-100 \% \mathrm{HRmax}=5$ ) and then summating the results.

The final criterion measure of internal TL used in this study was the LT zone method previously described by Impellizzeri et al. (17). This method involves multiplying the time spent in three HR zones (zone 1: below LT; zone 2: between LT and the AT; and zone 3: above AT) by a coefficient relative to each intensity zone $(k=1$ for zone $1, k=2$ for zone 2 , and $k=3$ for zone 3 ) and then summating the results. In addition to these HR-based methods, a measure of swimming distance (m) was used as the criterion measure of external TL.

\section{Statistical Analyses}

The relationship between session-RPE and previously used HR-based methods for measuring internal TL and measures of external TL were analyzed using the Pearson productmoment correlation. Statistical comparisons of session-RPE were also made between coaches and athletes during the investigation period. Training sessions were divided into those intended by coaches to be easy (RPE $<3$ ), moderate (RPE 3-5), and difficult (RPE > 5). A two-way analysis of variance (ANOVA) was then used to compare coaches' and athletes' perceptions for intensity, duration, and training load at each intensity level. Statistical significance was set at $p \leq$ 0.05. Effect size (ES) (Cohen d) was also calculated to analyze potential trends in the data comparing respective coaches' perceptions of planned exercise intensity with the swimmers' perceptions of actual exercise intensity. An ES of $<0.2$ was classified as a "trivial," $0.2-0.4$ as a "small," $0.4-0.7$ as a "moderate," and $>0.8$ as a "large" effect. SPSS statistical software package version 11.5 (SPSS Inc., Chicago, Ill) was used for all statistical calculations.

\section{Results}

Various HR, RPE, and distance measures were collected from 248 training sessions during a 3-month period. However, because of problems with obtaining clean HR data during swimming, only eight subjects completed the desired 20 training sessions in the time frame required. Therefore, individual correlations between HR, RPE, and distance methods for quantifying training load were based on 20 individual sessions, whereas group correlations were based on a total of $160 / 248$ training sessions. Correlations between session-RPE and HR-based methods were all significant $(p<0.01)$. Individual correlations are presented in Table 2.

Coaches' and athletes' evaluations of session-RPE (internal $\mathrm{TL}$, duration, and intensity) were also examined during the investigation period. There were moderate-to-strong correlations observed between coaches and athletes for training duration $(r=0.86, p<0.01)$, training intensity $(r=0.84, p<$ $0.01)$, and internal TL $(r=0.85, p<0.01)$. Repeatedmeasures ANOVA also revealed significant interaction effects for training intensity group $\times$ coach-athlete perception group for RPE $(F=6.458, p=0.003)$. The interaction effect indicates that the RPE difference depends on the exercise intensity at which the session is being completed. The estimated coach RPE was lower than the actual athlete RPE for low-intensity session, and the estimated coach RPE was higher than the actual athlete RPE for high-intensity sessions (see Figure 1). Additional ES analysis revealed moderate-sized effects at the low- $(\mathrm{d}=0.67)$ and highintensity $(\mathrm{d}=-0.50)$ sessions, with only trivial ES differences at moderate-intensity $(d=-0.14)$ sessions.

\section{Discussion}

The present investigation examined the ecological validity and practical application of the session-RPE method for quantifying training loads in swimmers. Our results are consistent with previous investigations that have shown a high correspondence between session-RPE and HR-based methods for evaluating internal TL in athletes $(12,13,15)$. In the present study, we found significant individual correlations between session-RPE and commonly used HR-based methods (e.g., TRIMP $[r=0.55-0.92]$, Edwards $[r=0.57-$ $0.91]$, and LT zone method [ $r=0.59-0.94]$ ) for quantifying internal TL $(11,18,19)$. However, the strength in the correlations between session-RPE and the HR-based methods were slightly lower than those reported in previous 


\begin{tabular}{|c|c|c|c|c|c|}
\hline Subjects & Distance & Session-RPE coach & Banister's TRIMP & Edward's TRIMP & LT zone \\
\hline S1 & 0.37 & 0.84 & 0.76 & 0.76 & 0.64 \\
\hline $\mathrm{S} 2$ & 0.76 & 0.88 & 0.79 & 0.82 & 0.73 \\
\hline S3 & 0.85 & 0.86 & 0.92 & 0.91 & 0.94 \\
\hline S4 & 0.70 & 0.93 & 0.63 & 0.63 & 0.78 \\
\hline S5 & 0.80 & 0.73 & 0.81 & 0.84 & 0.87 \\
\hline S6 & 0.35 & 0.91 & 0.56 & 0.57 & 0.71 \\
\hline S7 & 0.57 & 0.96 & 0.55 & 0.56 & 0.59 \\
\hline S8 & 0.81 & 0.94 & 0.92 & 0.91 & 0.91 \\
\hline Mean & 0.65 & 0.88 & 0.74 & 0.75 & 0.77 \\
\hline $\pm S D$ & 0.20 & 0.07 & 0.15 & 0.15 & 0.13 \\
\hline
\end{tabular}

investigations in endurance-based athletes $(r=0.75-0.90)$ (14) and slightly higher than those reported in young soccer players $(r=0.50-0.85)(17)$. These findings may be attributed to the type of training undertaken by the athletes in the present study (e.g., interval training).

Previous investigations have reported increases in RPE scores in subjects performing intermittent training compared with steady-state training where training sessions were matched for total work (10). These previous investigators have suggested that increases in RPE scores were attributable to increases in the anaerobic contribution to energy production during intermittent training. In accordance with these findings, Coutts et al. (8) recently have shown that the combination of $\mathrm{HR}$ and $\left[\mathrm{BLa}^{-}\right]$measures taken during smallsided soccer games was better related to RPE than HR and $\left[\mathrm{BLa}^{-}\right]$measures taken alone. These previous findings further support the argument that RPE may provide a valid global measure of training intensity during high-intensity, non-steady-state training. In the present investigation, the majority of training was performed by the athletes in the form of interval training. For example, depending on the focus of the session, the coaches often prescribed swim sessions that involved repeat swim efforts ranging from 25 to $800 \mathrm{~m}$. These commonly resulted in the swimmers having to undertake highly intermittent work. It is this intermittent work that may have been responsible for increases in RPE scores in relation to HR measures. We suggest that the global nature of RPE that is modulated by various forms of stress during exercise makes it a suitable practical tool for monitoring internal TL in swimmers.

The present results also show the session-RPE method to have the lowest correlation to distance measures for quantifying training load $(r=0.37-0.85)$. This finding was an expected result because distance measures do not take into account the intensity of the training session. For example, it would be far less stressful for a swimmer to perform $10 \times 100 \mathrm{~m}$ at an aerobic intensity than it would for the same swimmer to perform $10 \times 100 \mathrm{~m}$ at maximal intensity. This finding suggests that distance measures may provide a poor method for evaluating TL in athletes undertaking high-intensity training programs.

Although the session-RPE method may seem unsophisticated compared with HR-based approaches, both methods have been shown to be useful for evaluating internal TL in the majority of endurance-based sports. However, in agreement with previous investigations $(14,17)$, the present results suggest that the session-RPE method may be more sensitive than HR-based or distance measures for describing the response to training during high-intensity or intermittent exercise. Furthermore, because of technical problems, we were only able to obtain good HR data from 160/248 individual training sessions, demonstrating the level of difficulty in obtaining HR data during swimming. Therefore, given the importance of high-intensity exercise in swimming and the difficulties associated with collecting HR information in this environment, the session-RPE method may provide a more valid approach to monitoring internal $\mathrm{TL}$ in competitive swimmers.

The second purpose of this study was to examine the correspondence between athlete and coach perceptions of internal TL using the session-RPE method. No significant differences in internal TL were revealed between athletes and coaches during sessions designed to be easy (RPE $<3$ ), moderate (RPE 3-5), and difficult (RPE > 5). However, in agreement with previous research (16), the present results show a tendency for the athletes to report higher training intensities compared with coaches during sessions designed to be easy and lower training intensities compared with coaches during sessions designed to be difficult. These findings are consistent with other previous investigations that have 


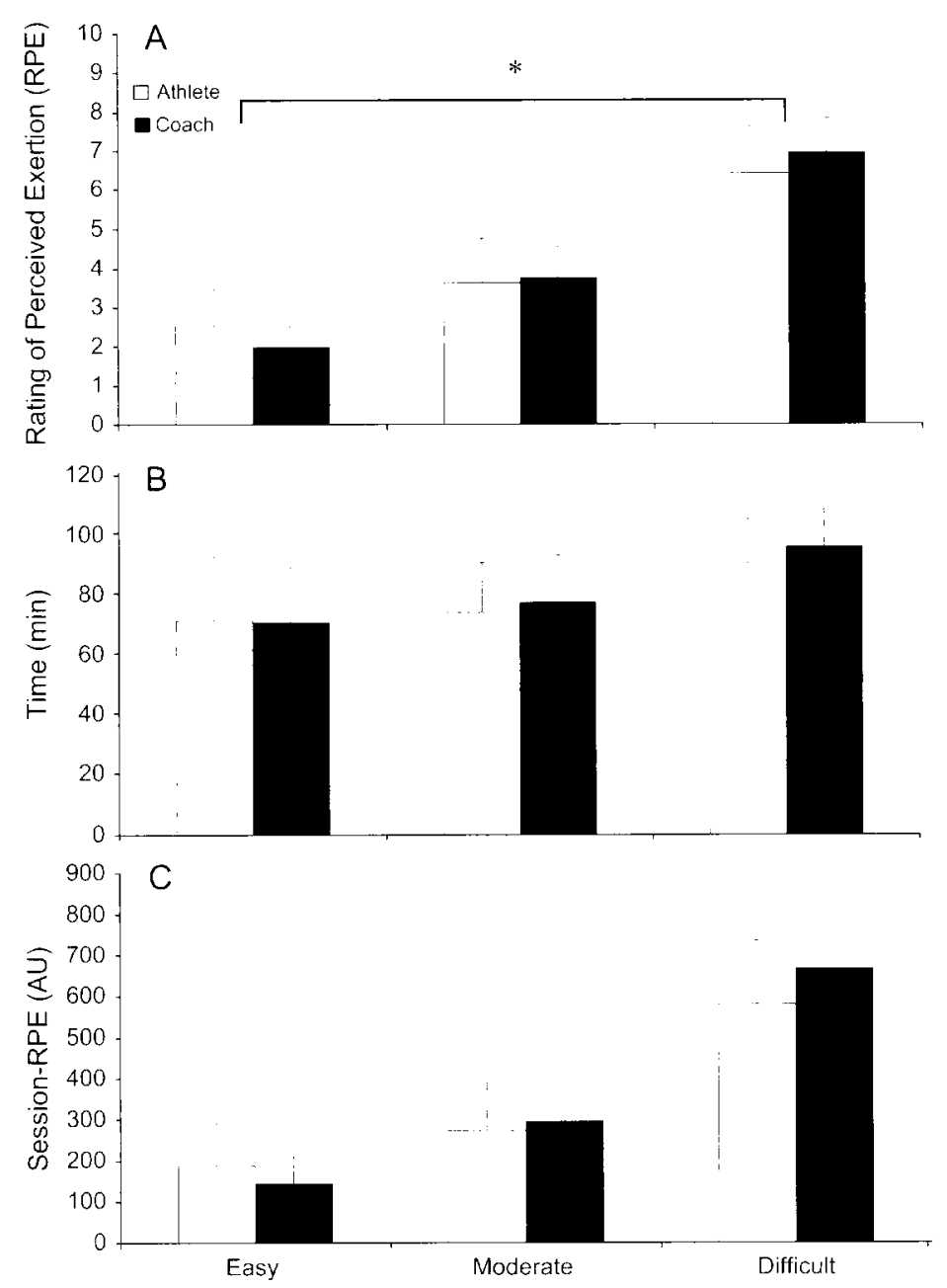

Figure 1. Comparison between athletes' and coaches' perceptions of intensity (A), duration (B), and internal TL (C) using the session-RPE method during easy, moderate, and difficult training sessions (mean $\pm S D$ ). * Significant interaction effect $(p<0.05)$

shown a mismatch between athletes' and coaches' perceptions of training intensity at low and high intensities (16).

A mismatch in perceived training intensity between athlete and coach has important implications for training athletes. This result demonstrates poor control of training variables and may place athletes at an increased risk of maladaptive training. It has previously been suggested that a decrease in the day-to-day variability in TL may increase the incidence of illness (12) and have a negative impact on performance (6). For example, Bruin et al. (6) observed symptoms associated with overtraining syndrome in race horses where "easy" days were increased in a program constructed on a "hard" day, "easy" day basis. The present results show how a system for monitoring internal TL such as the session-RPE method may improve control of training variables and provide a useful tool for quantifying the internal TL placed on athletes.

\section{Practical Applications}

At present, there are few valid and practical methods for monitoring internal TL during high-intensity, non-steadystate exercise such as swimming training. The session-RPE method may allow coaches to monitor the training process by quantifying the internal $\mathrm{TL}$ of an athlete using a single term (16). The benefits of using session-RPE include allowing coaches to evaluate and compare the training stress imposed on individual athletes during each component of the training program (e.g., swimming and dry land workouts). Furthermore, the use of RPE provides a cost-efficient, noninvasive, and reliable method for quantifying training intensity. The application of this method to swimming may also allow coaches to monitor training adaptations in individuals and to verify periodization strategies $(10,14,16)$. For example, an increased RPE to a regular standard work bout, during each training cycle (i.e., weekly) may be used as a guide for coaches to monitor for either increases in fatigue or reductions in fitness levels within individual athletes. Conversely, a reduction in RPE to these standard work bouts may indicate training adaptation. However, further research is required to validate the effectiveness of this method for monitoring changes in performance, fitness, and fatigue during swimming.

\section{References}

1. Achten, J and Jeukendrup, AE. Heart rate monitoring: applications and limitations. Sports Med 33: 517-538, 2003.

2. Banister, EW. Modeling elite athletic performance. In: Physiological Testing of Elite Athletes. Green, HJ, McDougal, JD, and Wenger, HA, eds. Champaign: Human Kinetics, 1991. pp. 403-424.

3. Banister, EW, Calvert, TW, Savage, MV, and Bach, T. A systems model of training for athletic performance. Aust J Sports Med Exerc Sci 7: 57-61, 1975.

4. Banister, EW and Fitz-Clark, JR. Plasticity of response to equal qualities of endurance training separated by non-training in humans. $J$ Therm Biol 5: 587-597, 1993. 
5. Borg, GAV, Hassmen, $P$, and Langerstrom, M. Perceived exertion in relation to heart rate and blood lactate during arm and leg exercise. Eur J Appl Physiol 65: 679-685, 1985.

6. Bruin, G, Kuipers, H, Keizer, HA, and Vander Vusse, GJ. Adaptation and overtraining in horses subjected to increasing training loads. $J$ Appl Physiol 76: 1908-1913,1994.

7. Coutts, AJ, Reaburn, PRJ, Murphy, AJ, Pine, MJ, and Impellizzeri, FM. Validity of the session-RPE method for determining training load in team sport athletes. $J$ Sci Med Sport 6: 525, 2003.

8. Coutts, AJ, Rampinini, E, Marcora, S, Castagna, C, and Impellizzeri, FM. Physiological correlates of perceived exertion during soccer-specific exercise. J Sci Med Sport December 6, 2007 [Epub ahead of print].

9. Day, M, McGuigan, MR, Brice, G, and Foster, C. Monitoring exercise intensity during resistance training using the session-RPE scale. J Strength Cond Res 18: 353-358, 2004.

10. Drust, B, Reilly, T, and Cable, NT. Physiological responses to laboratory-based soccer-specific intermittent and continuous exercise. J Sports Sci 18: 885-892, 2000.

11. Edwards, S. High performance training and racing. In: The Heart Rate Monitor Book. Edwards, S. ed. Sacramento: Feet Fleet Press, 1993. pp. 113-123.

12. Foster, C. Monitoring training in athletes with reference to overtraining syndrome. Med Sci Sports Exerc 30: 1164-1168, 1998.

13. Foster, C, Daines, E, Hector, L, Snyder, AC, and Welsh, R. Athletic performance in relation to training load. Wis Med J95: 370-374, 1996
14. Foster, C, Florhaug, JA, Franklin, J, Gottschall, L, Hrovatin, LA, Parker, S, Doleshal, P, and Dodge, C. A new approach to monitoring exercise training. J Strength Cond Res 15: 109-115, 2001.

15. Foster, C, Hector, LL, Welsh, R, Schrager, M, Green, MA, and Snyder, AC. Effects of specific versus cross-training on running performance. Eur J Appl Physiol 70: 367-372, 1995

16. Foster, C, Helmann, KM, Esten, PL, Brice, G, and Porcari, JP. Differences in perceptions of training by coaches and athletes. SASMA 8: 3-7, 2001.

17. Impellizzeri, FM, Rampinini, E, Coutts, AJ, Sassi, A, and Marcora, SM. The use of RPE-based training load in soccer. Med Sci Sports Exer 36: 1042-1047, 2004

18. Lucía, A, Hoyos, J, Santalla, A, Earnest, C, and Chicharro, JL. Tour de France versus Vuelta a Espana: which is harder? Med Sci Sports Exerc 35: 872-878, 2003

19. Morton, RH, Fitz-Clarke, JR, and Banister, EW. Modeling human performance in running. J Appl Physiol 69: 1171-1177, 1990.

20. Pyne, DB, Lee, $H$, and Swanwick, KM. Monitoring the lactate threshold in world-ranked swimmers. Med Sci Sports Exerc 33: 291-297, 2001

21. Svedah, $\mathrm{K}$ and MacIntosh, BR. Anaerobic threshold: the concept and methods of measurement. Can J Appl Physiol 28: $299-323,2003$

22. Viru, A and Viru, M. Nature of training effects. In: Exercise and Sport Science. Garret, WE, and Kirkendall, DT, eds. Philadelphia: Lippincott Williams and Wilkins, 2000. pp. 67-95. 